\title{
DLL1 Gene
}

National Cancer Institute

\section{Source}

National Cancer Institute. DLL1 Gene. NCI Thesaurus. Code C106582.

This gene is involved in notch signaling. 